\section{Mental Health Policy}

\section{Youth mental health now: Focus on prevention, intervention and outcomes}

\section{W0058}

\section{Prevention of substance abuse in youth: How social norms approach can help}

\section{E. Sönmez}

Department Of Psychiatry, Zonguldak Caycuma State Hospital, Istanbul, Turkey

doi: $10.1192 /$ j.eurpsy.2021.178

Drug and alcohol use in adolescence is a major global public health concern. Adolescence is the highest risk period for the initiation of drinking and substance use. Since the 90 s, a growing body of evidence has indicated the influence of peers' behaviours and attitudes in the development of youth tobacco, alcohol and drug use. Based on these studies, the social norms approach has been developed, mainly applying to the Western countries. The approach argues that how a student perceives his/her peers' health-related behaviours and attitudes (such as substance/alcohol use) does have an influence on his/her own behaviour, and negative behaviours are generally overestimated among peers. Correcting these misperceptions may contribute to the prevention of youth substance/alcohol use. The applicability and discussions on the social norms approach will be presented in this talk, with two example studies. Both studies are conducted in Turkey, which is ranked lowest in terms of alcohol use and related problems among World Health Organization Europe zone countries, despite and increasing trend in use over the past decades. In both university and high-school samples, we found that students' misperceptions about higher peer tobacco and alcohol use facilitated their own alcohol use. We conclude that targeting social norms may be part of a generalized preventive approach with regards to drug use and is of universal value. References: 1. SÖNMEZ, E. \& AKVARDAR, Y. 2015. A Social Norms Approach to Substance Abuse Prevention in Youth "The more I think you drink, the more I drink". Bağımlılık DergisiJournal of Dependence, 16, 86-94 (Turkish) 2. GÜNDÜZ, A., SAKARYA, S., SÖNMEZ, E., ÇELEBI, C., YÜCE, H. \& AKVARDAR, Y. 2019. Social norms regarding alcohol use and associated factors among university students in Turkey. Archives of Clinical Psychiatry (São Paulo), 46, 44-49.

Disclosure: No significant relationships.

Keywords: social norms; youth; alcohol use; prevention

\section{W0057}

Prodromal services for at-risk youth and their integration with existing programs: A "modular integration" model

\footnotetext{
A. Savic ${ }^{\star 1}$, D. Ostojic ${ }^{1}$, T. Jendricko ${ }^{2}$, P. Brecic ${ }^{3}$ and J. Vukojevic ${ }^{1}$

${ }^{1}$ Department Of Diagnostics And Intensive Care, University Psychiatric Hospital Vrapce, Zagreb, Croatia; ${ }^{2}$ Department Of Psychotherapy S, University Psychiatric Hospital Vrapče, Zagreb,
}

Croatia and ${ }^{3}$ Department For Affective Disorders, University Psychiatric Hospital Vrapče, Zagreb, Croatia

${ }^{\star}$ Corresponding Author.

doi: 10.1192/j.eurpsy.2021.179

Prodromal symptoms may precede onset of the psychotic disorders by years, and while we have instruments for identification of clinical high-risk (CHR), existing predictive models yield low specificity and fail in successfuly predicting transition to psychosis. This, along with the fact that we see a notable decline in transition rates, and the everlooming lack of resources, means CHR work is still often relegated to research settings. First-episode psychosis (FEP) unit of our institution offers both inpatient and outpatient programs, and while it is common having CHR services emerge from FEP frameworks, there is an inherent risk in drowning the diverse CHR population into very specific FEP interventions and settings, risking additional burden of stigma. Model our institution introduced includes: $\Rightarrow$ Nondependence on research funding; $\Rightarrow$ Flexible integration in available resources (e.g., FEP interventions, anxiety disorders interventions, substance-use programs); $\Rightarrow$ Dimensional approach with interdisciplinary assessment of capacities/needs non-reliant on supposed diagnosis; $\Rightarrow$ Embedded coordinating clinician ensuring "coordination" of CHR population "dispersed" among different programs; $\Rightarrow$ Collaboration with child psychiatrists allowing smoother transfer of at-risk youth; $\Rightarrow$ Recruitment through sensitized general practitioners and counseling services. No model of care, especially in area lacking conclusive predictive models, can be generalized to different health care systems, as practices and resources vary significantly. Nevertheless, the model presented makes a contribution to the care of $\mathrm{CHR}$ population, utilizing/integrating available resources, focusing on dimensional formulation of needs, and avoiding possible stigmatization. Furthermore, widely available CHR services, congruent with any research initiatives, might help us reach the necessary critical mass of data and experience needed for the final push towards clearer prediction models and treatment algorithms.

Disclosure: No significant relationships.

Keywords: clinical high-risk; prodromes; psychosis; early intervention

\section{W0058}

The impact of new psychosocial stresssors on the mental health of young people: Results from a national multicentric study in italy

\section{G. Sampogna}

Department Of Psychiatry, University of Campania "Luigi Vanvitelli”, Largo Madonna delle Grazie, Naples, Italy

doi: 10.1192/j.eurpsy.2021.180

The COVID-19 pandemic and its related containment measures, such as lockdown, is affecting mental health of the general population worldwide. This is an unprecedented event, which is influencing the health care, political, economic, and social welfare systems. Among Western countries, Italy has been one of the first severely hit by the pandemic in terms of number of cases and mortality rates. Therefore, on March 8, 2020, the Italian Prime Minister issued restrictive measures in order to limit the spread of the disease. During this period known as "Phase one" of the national health emergency, all not necessary activities have been closed, more than 
29,000 people have died and almost 100,000 people have been home-isolated, with strict lockdown measures. The COvid Mental hEalth Trial (COMET) network, including ten university Italian sites and the National Institute of Health, has promoted a national online survey in order to evaluate the impact of lockdown measures on the mental health of the Italian general population. The COMET survey reports data from a large sample of more than 20.000 people from Italian general population, showing that lockdown has had a detrimental impact on mental health, in terms of worsening of anxiety, depressive and stress symptoms. Findings from this study can be useful to inform national and international associations, policy makers and stakeholders on the importance to provide adequate support to the mental health of the general population.

Disclosure: No significant relationships.

Keywords: mental health; pandemic; Young People

\section{W0059}

Social media content analysis on twitter to explore public perceptions regarding pathological social withdrawal (hikikomori)

\section{Pereira-Sanchez ${ }^{\star 1,2}$ and M. Alvarez-Mon ${ }^{3}$}

${ }^{1}$ Psychiatry And Medical Psychology, Clinica Universidad de Navarra, Pamplona, Spain; ${ }^{2}$ Child And Adolescent Psychiatry, NYU Grossman School of Medicine, New York, United States of America and

${ }^{3}$ Department Of Psychiatry, University Hospital Infanta Leonor, Madrid, Spain

${ }^{\star}$ Corresponding Author.

doi: 10.1192/j.eurpsy.2021.181

Hikikomori is a form of severe social withdrawal, initially described in Japan and recently reported in other countries around the world. Individuals with hikikomori shut themselves in their homes with minimal interaction with society and little participation in school or the workforce. The nature of hikikomori makes the individuals suffering it a hard-to-reach population. While hikikomori was described in Japan much before the 'digital revolution' of the 2000 s, the internet, social media, and online gaming have radically changed the way people interact. This may be particularly true among hikikomori who spend much time online for entertainment or social interaction. Given this, the online world has been proposed as an accessible gateway to reach and support individuals with hikikomori. This talk will present and discuss the results of the Twitter-hikikomori international studies, conducted between 20182020 and led by Dr. Pereira-Sanchez, which employed social media mixed quantitative-method analyses to characterize the public conversations related to hikikomori on the social media platform Twitter in several Western languages and Japanese. As for the results, Twitter data provided evidence that hikikomori extends well beyond Japan examining, and showed that tweets in Japanese are more often are related to personal anecdotes, whereas tweets in Western languages are more often related to hikikomori as a medical issue. Apart from the results of the content analyses studies have been a proof of concept on the use of social media contents to investigate a phenomenon affecting a hard-to-reach population, which may inspire future online-based efforts to better support these populations.

Disclosure: No significant relationships.

Keywords: social withdrawal; social media; Hikikomori; twitter

\section{Clinical/Therapeutic}

Medication deprescribing in elderly patients with
mental disorders: Why, when, and how?

\section{W0060}

\section{Different general strategies for deprescribing in real clinical settings: From lists to collaborative care}

\section{Stuhec ${ }^{\star 1,2}$}

${ }^{1}$ Clinical Pharmacy \& Pharmacology, University of Maribor, Medical Faculty, Maribor, Slovenia and ${ }^{2}$ Clinical Pharmacy, University of Ljubljana, Faculty of Pharmacy \& Ormoz Psychiatric Hospital, Ormoz, Slovenia

${ }^{\star}$ Corresponding Author. doi: 10.1192/j.eurpsy.2021.182

Most elderly patients with mental disorders are treated with polypharmacy (e.g., five or more medications), and they are receiving medications that are potentially inappropriate for elderly patients (e.g., PIMs). These aspects are often excluded in the clinical guidelines, meta-analyses, and randomized controlled trials but are very important for prudent prescribing in daily practice. The most robust approach to reducing irrational polypharmacy, PIMs, and other medications-related problems in this population is a careful deprescribing process. It is the process of tapering, withdrawing, discontinuing, or stopping medications. There are some tools available to help in the deprescribing process in clinical practice, including different medication lists (e.g., Beers criteria, STOPP/START, and guidelines) and collaborative care, including clinical pharmacist or pharmacologist. Medication lists have been used in clinical trials and guidelines, where Beers criteria are used predominantly in the U.S. and Priscus list in Europe. A collaborative care approach, including a clinical pharmacist, has been established only in some countries (e.g., USA, UK \& Slovenia). The results are positive with a decrease of PIMs, polypharmacy, and an increase in the patients' quality of life. The participants will learn the general deprescribing processes supported by the evidence-based data and real clinical pharmacological tools useful for daily practice.

Disclosure: No significant relationships.

Keywords: Psychopharmacology; Collaborative care; Deprescribing; Real Clinical Setting

\section{W0061}

\section{Clinical aspects of deprescribing process in affective disorders}

\section{F. Bouckaert}

Department Of Geriatric Psychiatry, University Psychiatric Center KU Leuven \& Laboratory for Translational Neuropsychiatry, Leuven, Belgium doi: 10.1192/j.eurpsy.2021.183

Although depression in the elderly is often underdiagnosed and undertreated, some data show that next to this potential underuse, antidepressant prescriptions may also be overused and prescribed inappropriately. These potentially overused and inappropriate prescriptions of antidepressants are often related to polypharmacy, comorbidity and increased mortality. Deprescribing is the planned 